\title{
Glucagon-like peptide 1 receptor agonist ameliorates the insulin resistance function of islet $\beta$ cells via the activation of PDX-1/JAK signaling transduction in C57/BL6 mice with high-fat diet-induced diabetes
}

\author{
TAO HAO ${ }^{1,2}$, HONGTAO ZHANG $^{3}$, SHEYU LI $^{1}$ and HAOMING TIAN ${ }^{1}$ \\ ${ }^{1}$ Department of Endocrinology and Metabolism, West China Hospital, Sichuan University, Chengdu, Sichuan 610041; \\ ${ }^{2}$ Department of Endocrinology and Metabolism, The First People's Hospital of Dujiangyan, Dujiangyan, \\ Sichuan 611830; ${ }^{3}$ Shaanxi Provincial People's Hospital, Xi'an, Shaanxi 710068, P.R. China
}

Received September 28, 2016; Accepted February 21, 2017

DOI: $10.3892 /$ ijmm.2017.2910

\begin{abstract}
Long-term exposure to a high-fat diet (HFD) causes glucotoxicity and lipotoxicity in islet $\beta$ cells and leads to the development of metabolic dysfunctions. Reductions in pancreatic and duodenal homeobox-1 (PDX-1) expression have been shown to induce type 2 diabetes mellitus by causing impairments to islet $\beta$ cells. Glucagon-like peptide 1 (GLP-1) treatment reduces endogenous insulin resistance in HFD-induced type 2 diabetes mellitus. In the present study, the underlying mechanism by which GLP-1 exerts its function in type 2 diabetes mellitus was investigated. The effect of liraglutide (GLP-1 receptor agonist) administration on glucose tolerance, insulin release, and glucose-dependent insulinotropic polypeptide level was detected in a HFD-induced diabetes C57/BL6 mouse model. Moreover, the role of liraglutide administration on the activity of PDX-1 was quantified to demonstrate the association between the two indicators. The results showed that administration of liraglutide could ameliorate the impairments to $\beta$ cells due to HFD consumption. Liraglutide restored the insulin capacity and stimulated glucose disposal by improving the function and increasing the number of islet $\beta$ cells. Furthermore, the hyperplasia and redundant function of islet $\alpha$ cells were inhibited by liraglutide treatment as well. At the molecular level, administration of liraglutide induced the expression of PDX-1, MafA, p-JAK2 and p-Stat3 in HFD model to relatively normal levels. It was suggested that the effect of liraglutide-induced activation of GLP-1 was exerted via activation of PDX-1 rather than its function in
\end{abstract}

Correspondence to: Dr Haoming Tian, Department of Endocrinology and Metabolism, West China Hospital, Sichuan University, 37 Guoxue Road, Chengdu, Sichuan 610041, P.R. China

E-mail: hmtian999@126.com

Key words: glucagon-like peptide 1, liraglutide, pancreatic and duodenal homeobox-1, type 2 diabetes, islet $\beta$ cells, signal decreasing body weight. The study demonstrated that GLP-1 played an essential role in type 2 diabetes mellitus.

\section{Introduction}

Obesity and its related metabolic disorder, non-insulin-dependent type 2 diabetes mellitus, are prevalent and affect more than 150 million people in Western countries (1). The incidence of type 2 diabetes mellitus continues to increase and has reached a proportion at an epidemic level which severely affects public healthcare and costs both human lives and financial cost $(2,3)$. Generally, the high-fat content in typical Western diets has been recognized as an important factor contributing to obesity and its related insulin resistance in type 2 diabetes mellitus $(4,5)$. Previous studies indicate that defective insulin secretion in type 2 diabetes mellitus is caused by islet $\beta$ cell dysfunction and reduced islet $\beta$ cell mass (6-8). Long-term hyperglycemia or/and hyperlipidemia due to a high-fat diet (HFD) cause glucotoxicity and lipotoxicity in $\beta$ pancreatic cells further leading to the development of metabolic dysfunctions (6-8). Thus, development of therapies targeting the improvement of islet $\beta$ cell function has become a central subject in the field related to the treatment of type 2 diabetes mellitus.

Owing to the rapid progress of genetics and omics techniques, numerous discoveries of molecular compounds influencing the function and development of islet $\beta$ cells were elucidated (9-12). Among all these regulators, PDX-1 is a pancreatic and duodenal homeobox-1 transcription factor that modulates the development and differentiation of pancreatic islet $\beta$ cells (13). It has been confirmed that both genetic and acquired reductions in PDX-1 expression in humans and in animal models have been involved in the causes of the onset of type 2 diabetes mellitus, hepatic diabetes mellitus, and dysfunction in $\beta$ pancreatic cells (14-16). In adults, expression of PDX-1 is maintained in the duodenal epithelium and in insulin-secreting islet $\beta$ cells, where it may activate transcription of insulin genes $(17,18)$. In mouse embryos, PDX-1 expression precedes insulin and glucagon expression (12). As reported by Stoffers et al focusing on intrauterine growth 
retardation due to diseases in adulthood, mRNA levels of PDX-1 are inhibited in more than 50\% of IUGR fetuses (19). Therefore, upregulation of PDX-1 levels in islet $\beta$ cells may lead to the improvement of cell function and facilitate the treatment of type 2 diabetes mellitus.

Glucagon-like peptide 1 (GLP-1) is a peptide of 30 amino acids and is produced by L cells of the intestinal mucosa (20). The molecule responds to food intake and plays a major role in the control of postprandial metabolism by augmenting nutrient-induced insulin release, inhibiting glucagon secretion, and reducing endogenous glucose production $(21,22)$. Studies in normal weight cases showed that peripheral administration of GLP-1 decreased food intake and suppressed appetite $(23,24)$. A study based on mice fed with an HFD also revealed that GPL-1 treatment reduced endogenous insulin resistance via activation of central GLP-1 receptors (25). Although multiple studies demonstrated the beneficial effects of GLP-1 on HFD-induced type 2 diabetes mellitus are validated by multiple studies, the underlying mechanism involved in this treatment process is only partially revealed. Considering the key role of PDX-1 in the onset of type 2 diabetes mellitus and dysfunction of islet $\beta$ cells, we conducted a comprehensive study to assess the interaction between GLP-1 and PDX-1, which may provide novel insight into the treatment strategies for type 2 diabetes mellitus.

In the present study, C57/BL6 mice were fed with a HFD to mimic the type 2 diabetes mellitus condition in vivo. Then the effect of GLP-1 administration on glucose tolerance, insulin release, and glucose-dependent insulinotropic polypeptide (GIP) level was detected for assessment of islet $\beta$ cell function. Moreover, the effect of GLP-1 administration on the activation of PDX-1-related signaling transduction was also quantified to profile the interaction between the two indicators.

\section{Materials and methods}

Chemicals and animals. Liraglutide (GLP-1 receptor agonist) (cat. no. J20110020) and human insulin Novolin (cat. no. J20120026) were both purchased from Novo Nordisk (Oslo, Norway). Eight-week-old C57/BL6 mice were provided by the Laboratory Animal Center, West China Hospital, Sichuan West China School of Medicine (Chengdu, China) and maintained in cages at room temperature $\left(20-25^{\circ} \mathrm{C}\right)$ with a constant humidity $(55 \pm 5 \%)$ with free access to food and water in a 12:12-h light/dark cycle. All animal experiments were conducted in accordance with the Institutional Animal Ethics Committee and Animal Care Guidelines for the Care and Use of Laboratory Animals of Sichuan West China School of Medicine.

HFD treatment and animal grouping. Thirty-two mice were randomly divided into four groups (8 for each group) with different administrations: i) control group, mice fed with standard diet $(7.8 \%$ fat, $41.9 \%$ protein and $50.3 \%$ carbohydrate, total energy of $2.18 \mathrm{kcal} / \mathrm{g}$ ) for 12 weeks; ii) HFD group, mice fed with HFD (61\% fat, $15 \%$ protein and $25 \%$ carbohydrate, $0.2 \%$ cholesterol, total energy of $5.28 \mathrm{kcal} / \mathrm{g})+$ high glucose water ( $2 \%$ fructopyranose plus $2.5 \%$ glucose) ad libitum for 12 weeks; iii) liraglutide group, mice fed with HFD (61\% fat, $15 \%$ protein and $25 \%$ carbohydrate, $0.2 \%$ cholesterol, total energy of $5.28 \mathrm{kcal} / \mathrm{g})$ + high glucose water ( $2 \%$ fructopyranose plus $2.5 \%$ glucose) ad libitum for 12 weeks and GPL-1 (0.6 mg/ $\mathrm{kg}$ body weight/day) was subcutaneously injected into mice from the 8th week of the experiment; iv) liraglutide paired group, mice in this group were fed with an HFD but were calorically restricted to follow the weight trajectory of mice in the liraglutide group. The group was set up to eliminate the possibility that the effect of liraglutide-induced GLP-1 activation was exerted through the influence on the body weight of the treated mice. Body weight of mice in each group was measured every two weeks until the completion of the different administrations.

Oral glucose tolerance test (OGTT) and insulin release test. Upon completion of the 12-week treatment, mice ingested a solution containing $2 \mathrm{~g} / \mathrm{kg}$ of dextrose after a 5-h overnight fast. Thereafter, tail venous blood samples of mice were obtained with a sterile syringe at $0,30,60,90$ and 120 min for determination of plasma glucose using the Glucose Oxidase Activity Assay kit (cat. no. MAK097; Sigma-Aldrich, St. Louis, MO, USA) according to the manufacturer's instructions. The plasma insulin levels at corresponding time-points were measured using Mouse Insulin ELISA kit (cat. no. ab100578) according to the manufacturer's instructions.

Hyperinsulinemic euglycemic clamp assay. For the hyperinsulinemic euglycemic clamp experiment, mice were previously fasted for $16 \mathrm{~h}$ and then sedated with $1 \%$ pentobarbital (cat. no. P3761; Sigma-Aldrich). Basal blood glucose (BBG) levels of mice in the different groups were measured with blood sampled from the tail vein using the Glucose Oxidase Activity Assay kit (cat. no. MAK097; Sigma-Aldrich) according to the manufacturer's instructions. Then a T-branch pipe was placed at the jugular vein of the mice. Insulin $(25 \mathrm{mU} / \mathrm{kg} / \mathrm{min})$ and glucose [basal glucose infusion rate (GIR), $360 \mathrm{mg} / \mathrm{kg} / \mathrm{h}$ ] were continuously dripped into the mice through one branch of the T-branch pipe for $120 \mathrm{~min}$. Blood glucose content was measured every $10 \mathrm{~min}$ until a level of BBG $\pm 0.05 \mathrm{mmol} / \mathrm{l}$ (stable blood glucose). During the experimental, GIR was adjusted according to the stable blood glucose. When the blood glucose level was lower than $5 \mathrm{mmol} / \mathrm{l}$, infusion of insulin was stopped.

Fasting insulin and GIP level. Upon completion of hyperinsulinemic euglycemic clamp assay, mice were fasted for $16 \mathrm{~h}$ to determine the fasting insulin and GIP level. Insulin level was determined using the Mouse Insulin ELISA kit according to the manufacturer's instructions. The GIP level was also determined using the GIP ELISA kit (cat. no. RAB0209; Sigma-Aldrich) according to the manufacturer's instructions. Upon completion of measurement of fasting insulin and GIP levels, the mice were sacrificed using air embolism methods and their pancreatic tissues were harvested and preserved at $-80^{\circ} \mathrm{C}$ for further histological and molecular analyses.

Hematoxylin and eosin (H\&E) staining and TUNEL staining. The histological changes in sections of the pancreas from the different groups were observed using routine $\mathrm{H} \& \mathrm{E}$ staining and the results were detected under a microscope at x20 magnification. Cell apoptotic rates in the pancreatic tissues were determined with TUNEL staining using DeadEnd $^{\text {TM }}$ Fluorometric TUNEL system (cat. no. G3250; Promega, Madison, WI, USA) and the results were detected using fluorescent microscopy at x200 magnification. 
Immunofluorescent microscopy. Expression of the insulin antibody and glucagon antibody in the pancreatic tissues was detected with immunofluorescent microscopy. Briefly, the treated cells were seeded into 24-well chambers, washed with phosphate-buffered saline (PBS), and fixed with $4 \%$ paraformaldehyde for $15 \mathrm{~min}$. Then the cells were permeabilized with $0.5 \%$ Triton X-100 for $30 \mathrm{~min}$. After being washed with PBS for three cycles, the cells were blocked in $10 \%$ goat serum for $15 \mathrm{~min}$. Primary rabbit polyclonal antibodies to insulin (1:200, cat. no. EPR17359) and glucagon (1:200, cat. no. EP3070) (both from Abcam, Cambridge, MA, USA) were then added and the cells were incubated overnight at $4^{\circ} \mathrm{C}$ in $1 \%$ goat serum. Staining was performed by incubating the cells with fluorescein isothiocyanate secondary antibody for $1 \mathrm{~h}$. After incubation with the secondary antibody $(1: 1,000)$, the cells were washed and then stained with 4',6-diamidino2-phenylindole (DAPI) for $5 \mathrm{~min}$ at room temperature. After extensive washing with PBS, the slides were fixed and imaged with fluorescent microscopy at x40 magnification.

Reverse transcription-quantitative PCR (RT-qPCR). For RT-qPCR detection, whole RNA in pancreatic tissues from the different groups was extracted using RNA Simple Total RNA kit according to the manufacturer's instructions (cat. no. DP419; Tiangen Biotech Co., Ltd., Beijing, China). $\beta$-actin was selected as the internal reference gene. Then the whole RNA was reversely transcribed to cDNA templates using Super M-MLV reverse transcriptase (cat. no. RP6502; BioTeke Corporation, Beijing, China). The final qPCR reaction mixture of volume $20 \mu 1$ consisted of $10 \mu 1$ of SYBR-Green Master Mix, $0.5 \mu 1$ of each primer (PDX-1 forward, 5'-CCCCAGTTTACAAGCTC GCT-3' and reverse, 5'-CTCGGTTCCATTCGGGAAAGG-3'; MafA forward, 5'-AGGAGGAGGTCATCCGACTG-3' and reverse, 5'-CTTCTCGCTCTCCAGAATGTG-3'; $\beta$-actin forward, 5'-CATTGCTGACAGGATGCAGA-3' and reverse, 5'-CTGCTGGAAGGTGGACAGTGA-3'), $1 \mu \mathrm{l}$ of the cDNA template, and $8 \mu \mathrm{l}$ of RNase-free $\mathrm{H}_{2} \mathrm{O}$. Amplification parameters were as follows: denaturation at $94^{\circ} \mathrm{C}$ for $2 \mathrm{~min}$, followed by 40 cycles at $94^{\circ} \mathrm{C}$ for $20 \mathrm{sec}, 58^{\circ} \mathrm{C}$ for $20 \mathrm{sec}$ and $72^{\circ} \mathrm{C}$ for $20 \mathrm{sec}$. Relative expression levels of the targeted molecules were calculated with DataAssist software version 3.0 (Applied Biosystems; Life Technologies, Carlsbad, CA, USA) according to the $2^{-\Delta \Delta c q}$ method.

Western blotting. The whole protein product in the different groups was extracted using the Total Protein Extraction kit according to the manufacturer's instructions (cat. no. WLA019; Wanleibio, Shenyang, China). GAPDH was used as the internal reference. Concentration of protein samples was determined using the BCA method. Twenty microliters of protein $(40 \mu \mathrm{g})$ was subject to $10 \%$ sodium dodecyl sulfate-polyacrylamide gel electrophoresis (SDS-PAGE). Targeted proteins were transferred onto polyvinylidene difluoride (PVDF) sheets and the membranes were washed with TTBS for $5 \mathrm{~min}$ before incubation in skim milk powder solution for $1 \mathrm{~h}$. Primary antibodies against PDX-1 (1:1,000, cat. no. 5679P; Cell Signaling Technology, Inc., Danvers, MA, USA), MafA (1:1,000, cat. no. ab26405; Abcam) (26), p-JAK2 (1:2,000, cat. no. 8082) (30), p-STAT3 (1:5,000, cat.no. 9145) (27) and Bax (1:1,500, cat. no. BS2538) (all from Cell Signaling Technology,
Inc., Boston, MA, USA), Bcl-2 (1:1,000, cat. no. BS1511) and GAPDH (1:10,000, cat. no. ab9485) (both from Abcam) were incubated with membranes at $4^{\circ} \mathrm{C}$ overnight. After four washes using TTBS, secondary HRP goat anti-rabbit IgG antibodies (1:20,000, cat. no. BA1054; Boster Biological Technology, Ltd., Wuhan, China) were added into the mixture and incubated with the membranes for $45 \mathrm{~min}$ at $37^{\circ} \mathrm{C}$. After an additional six washes with TTBS, the blots were developed using Beyo ECL Plus reagent and the results were detected in the gel imaging system. The relative expression levels of BDNF in the different samples were calculated with Gel-Pro Analyzer (Media Cybernetics Inc., Rockville, MD, USA).

Statistical analysis. All the data are expressed as the mean \pm SD. Two-group data were compared using the Student's t-test or paired Student's t-test. Significance was accepted at $\mathrm{P}<0.05$. All the statistical analysis and graph manipulation were conducted using GraphPad Prism 6 (GraphPad Software, Inc., San Diego, CA, USA).

\section{Results}

Administration of liraglutide improved the HFD-induced insulin resistance in the C57/BL6 mice. The body weight and energy intake changes in the different groups during 12 weeks are shown in Fig. 1. HFD consumption increased the body weight of the mice in the HFD group compared with control and liraglutide groups $(\mathrm{P}<0.05)$, while administration of liraglutide confounded the effect of the HFD on body weight changes in the mice from the 9 th week of the experiment $(\mathrm{P}<0.05)$ (Fig. 1).

To assess the insulin resistance induced by the HFD, a hyperinsulinemic euglycemic clamp assay was conducted. As shown in Fig. 2, the GIR value in the HFD group was lower than the other three groups (representing stronger insulin resistance), and the difference was statistically significant $(\mathrm{P}<0.05)$. Administration of liraglutide significantly increased the GIR value compared with GIR values in the HFD and liraglutide paired groups (representing weaker insulin resistance). However, GIR values in the liraglutide group were even significantly higher than those in the control group $(\mathrm{P}<0.05)$ (Fig. 2). Taken together, the results of the hyperinsulinemic euglycemic clamp assay showed that the insulin resistance induced by HFD could be ameliorated by treatment of liraglutide, but the optimal amount for practical application of liraglutide should be further assessed. Furthermore, considering no significant difference could be detected between the HFD and liraglutide paired groups, it was concluded that the insulin resistance induced by HFD was attributed to the composition of the diet instead of the amount of the diet.

Administration of liraglutide ameliorates the HFD-induced islet $\beta$ cell dysfunction and delays insulin release in the C57/BL6 mice. Based on the results of the OGTT assay, HFD treatment decreased the glucose tolerance in the experimental mice (Fig. 3A) with the highest plasma insulin level in all four groups (Fig. 3B), which indicated impairments in islet $\beta$ cells and a delayed release of insulin. However, treatment with liraglutide reversed the abnormal glucose tolerance induced by the HFD, inferring a pancreas-protecting effect of liraglutide (Fig. 3A). 
A

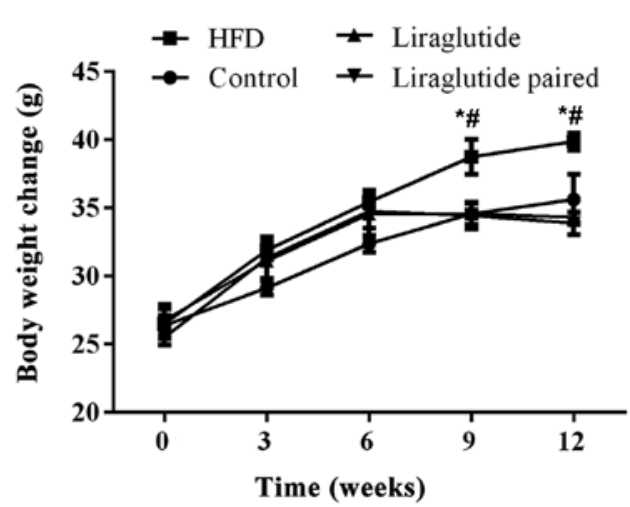

B

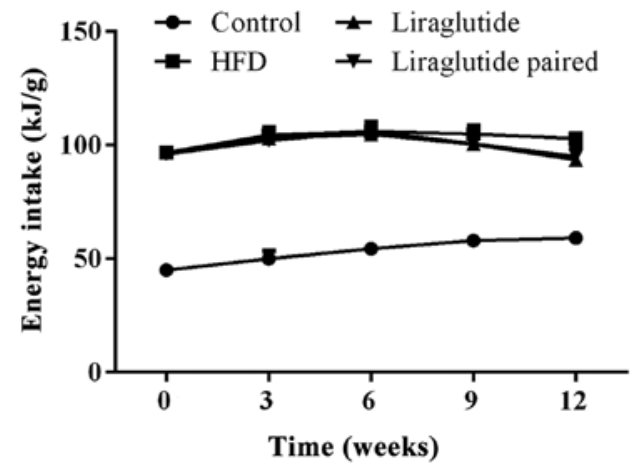

Figure 1. Body weight change and energy intake of mice under different treatments. (A) Body weight changes of the mice in the different groups during 12 weeks. (B) Energy intake of the mice in the different groups during 12 weeks. ${ }^{*} \mathrm{P}<0.05$, compared to the control group; ${ }^{~} \mathrm{P}<0.05$, compared to the liraglutide group.

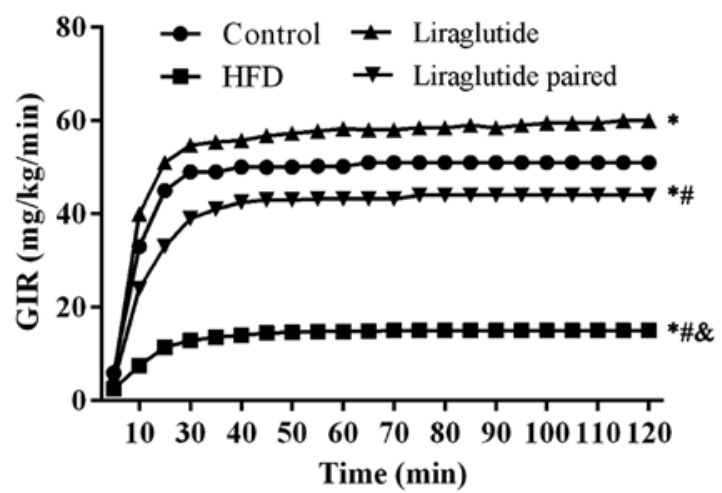

Figure 2. Administration of liraglutide improved the high-fat diet(HFD)-induced insulin resistance in mice. The glucose infusion rate (GIR) value in the group treated with HFD was significantly reduced from 10 to $120 \mathrm{~min}$, compared to that in the other 3 groups. Liraglutide statistically increased the level of GIR, compared to that in the control group. The liraglutide paired group showed a reduction of GIR compared to the liraglutide group. ${ }^{*} \mathrm{P}<0.05$, compared to the control group; ${ }^{\#} \mathrm{P}<0.05$, compared to the liraglutide group; ${ }^{\&} \mathrm{P}<0.05$, compared to the liraglutide paired group, from the time point of 10 to $120 \mathrm{~min}$.

Measurement of fasting insulin also confirmed damage to islet $\beta$ cell function (Fig. 4A). HFD markedly increased the fasting plasma insulin level when compared with control group at the last sampling point, and the differences were both statistically significant $(\mathrm{P}<0.05)$. The treatment of HFD also significantly increased the plasma GIP level in the HFD and
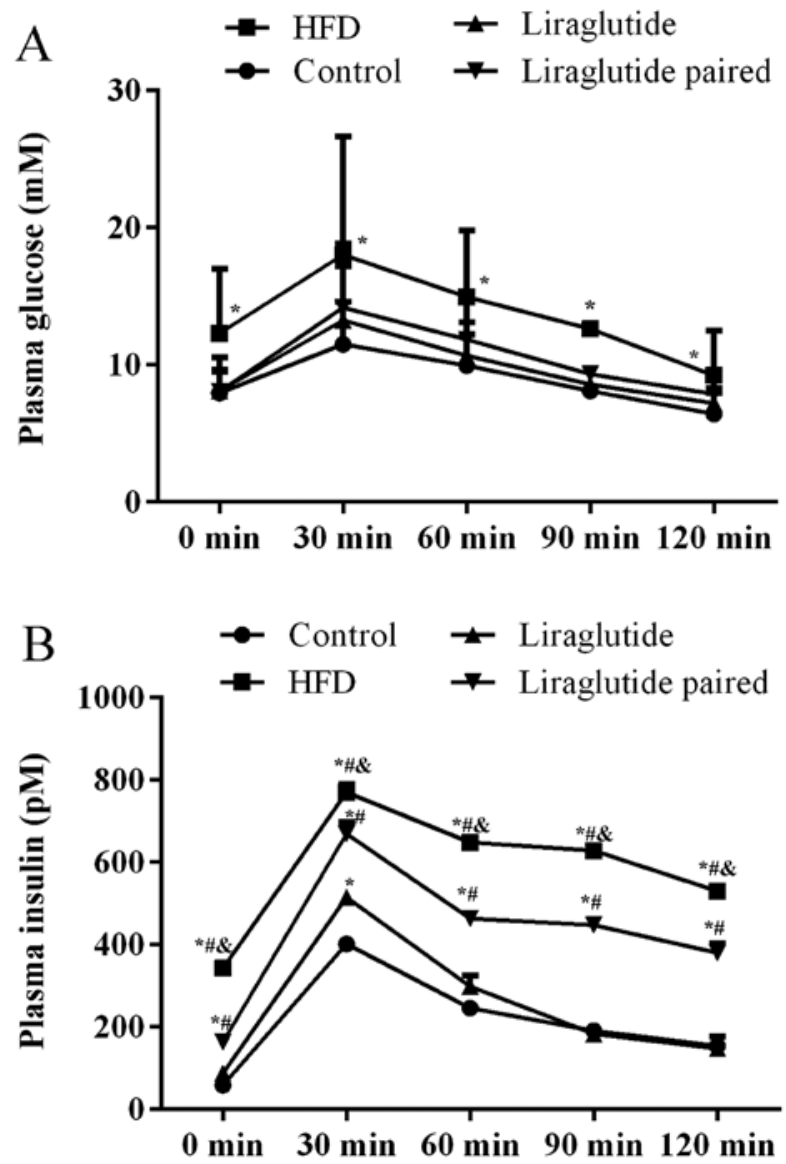

Figure 3. Administration of liraglutide improves the insulin tolerance in high-fat diet (HFD)-induced diabetes mice. (A) Levels of plasma glucose as determined by oral glucose tolerance test (OGTT) assay. Administration of liraglutide decreased the level of plasma glucose induced by HFD. ${ }^{*} \mathrm{P}<0.05$, compared with the other groups. (B) Levels of plasma insulin. Administration of liraglutide decreased the level of plasma insulin induced by HFD. ${ }^{*} \mathrm{P}<0.05$, compared to the control group; ${ }^{\#} \mathrm{P}<0.05$, compared to the liraglutide group; ${ }^{\&} \mathrm{P}<0.05$, compared to the liraglutide paired group.

liraglutide paired groups when compared with the other two groups (Fig. 4B) $(\mathrm{P}<0.05)$, representing augmentation of islet $\alpha$ cell function in the model animals. The increasing level of fasting insulin and GIP level in the HFD and liraglutide paired groups may be the key reason that HFD induced insulin resistance in mice. Similarly to the results of the OGTT assay, liraglutide administration confound the effect of HFD on the abnormal function of the pancreas (Fig. 4).

Additionally, it was shown by the above data that although mice in the liraglutide paired group exhibited a similar changing pattern in body weight to the liraglutide group, the induced insulin, fasting insulin, and fasting GIP levels were all significantly different from those in the liraglutide group $(\mathrm{P}<0.05)$, which evidently confirmed our hypothesis that the effect of liraglutide on the pancreas was not attributed to its effect on body weight.

Administration of liraglutide inhibits HFD-induced islet $\beta$ cell apoptosis and islet $\alpha$ cell hyperplasia in C57/BL6 mice. Following H\&E staining, nuclei in pancreatic tissues were stained blue and the cytoplasm was stained red. The degree of staining varied with ages of the cells. Based on the above criteria, a comparison between control and HFD 

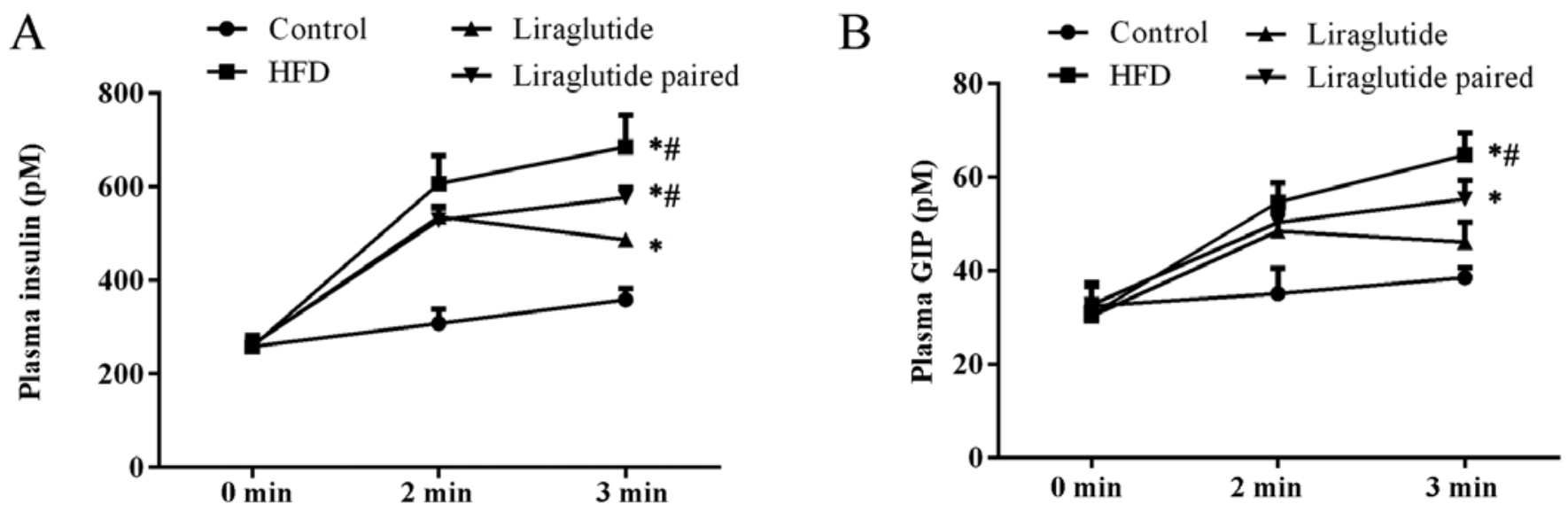

Figure 4. Administration of liraglutide decreases the fasting insulin and glucose-dependent insulinotropic polypeptide (GIP) levels in high-fat diet (HFD)-induced diabetes mice. (A) Administration of liraglutide reduced the level of fasting insulin in the HFD-induced diabetes mice. (B) Administration of liraglutide reduced the level of fasting GIP in the HFD-induced diabetes mice. ${ }^{*} \mathrm{P}<0.05$, compared to the control group; ${ }^{\#} \mathrm{P}<0.05$, compared to the liraglutide group.
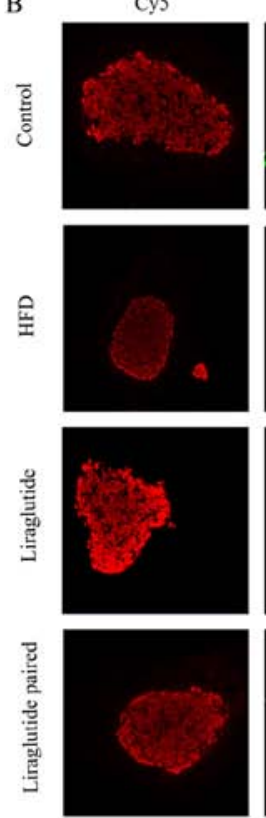

FITC
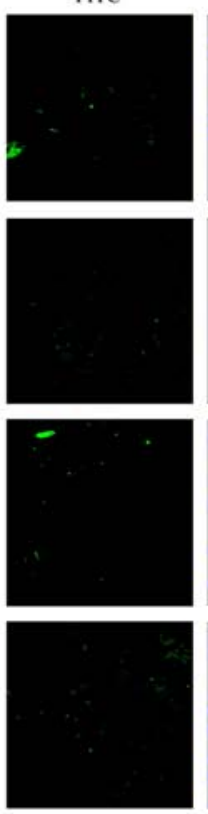

A

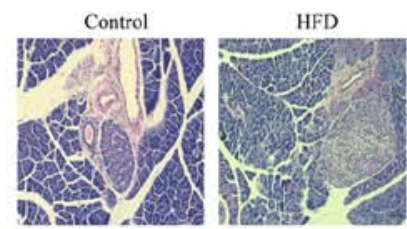

DAPI
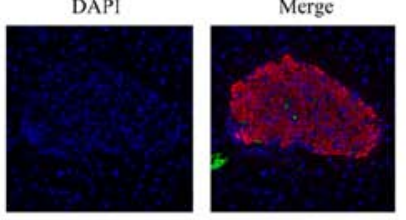

C
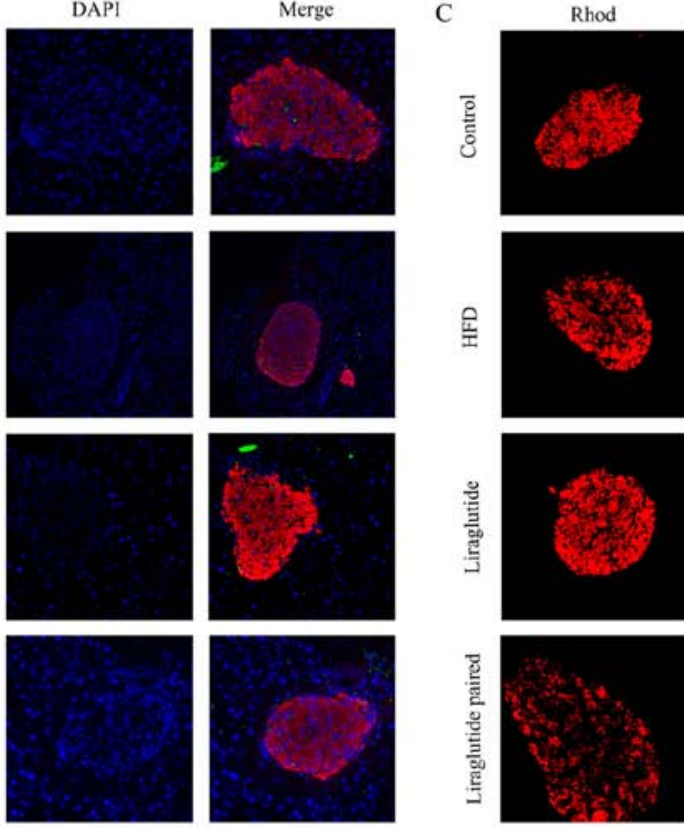
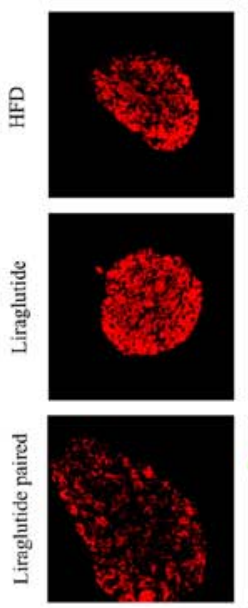

Liraglutide

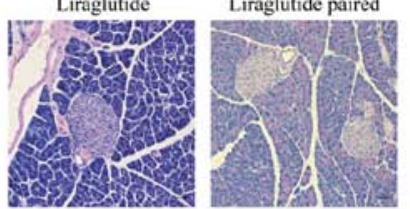

Cy5
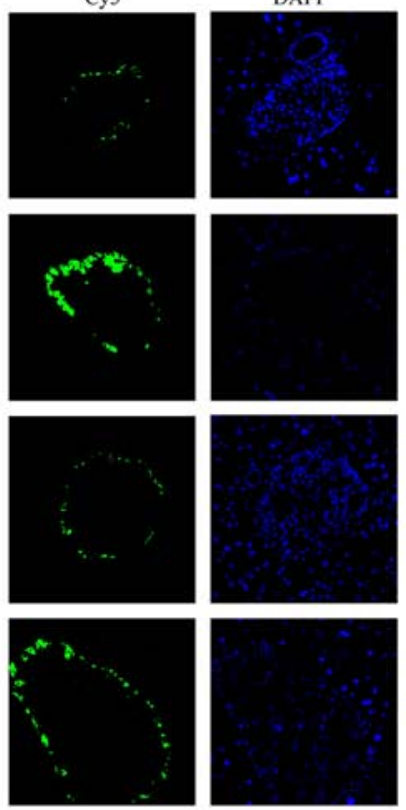
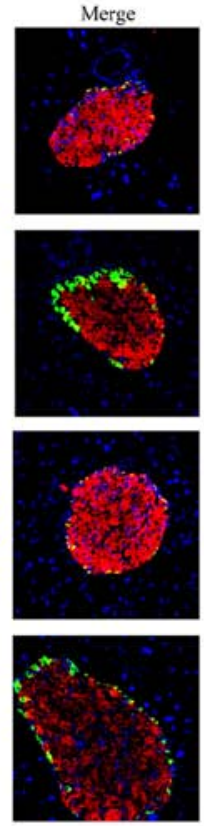

Figure 5. Administration of liraglutide improves the structure of pancreas islets, inhibits apoptosis of islet $\beta$ cells, and suppresses hyperplasia of islet $\alpha$ cells. (A) Representative images of H\&E staining at x20 magnification. In the high-fat diet (HFD) and liraglutide paired groups, volumes of pancreas islets were obviously increased and the infiltration of inflammatory cells could be clearly observed in the HFD and liraglutide paired groups. Administration with liraglutide alleviated the damage to the pancreas in that the numbers of aged cells decreased in the liraglutide group compared with the HFD and liraglutide paired groups and no obvious proliferation of pancreas islets could be detected in the liraglutide group. (B) Representative images of TUNEL staining at x200 magnification. FITC (TUNEL-positive) stained green, Cy5 (insulin-positive) stained red and DAPI stained blue. (C) Representative images of immunofluorescent detection at x40 magnification. Islet $\beta$ cells (stained red) were reduced in the HFD and liraglutide paired groups, and administration of liraglutide increased the number of islet $\beta$ cells to a level compared with the control group. The number of islet $\alpha$ cells (stained green) was clearly increased by HFD treatment, and administration of liraglutide inhibited the proliferation of islet $\alpha$ cells.

groups revealed severe injury due to HFD. In the HFD and liraglutide paired groups, the volumes of pancreas islet obviously increased and the infiltration of inflammatory cells was clearly observed (Fig. 5A). Administration with liraglutide alleviated the damage in which numbers of aged cells decreased in the liraglutide compared with that in 


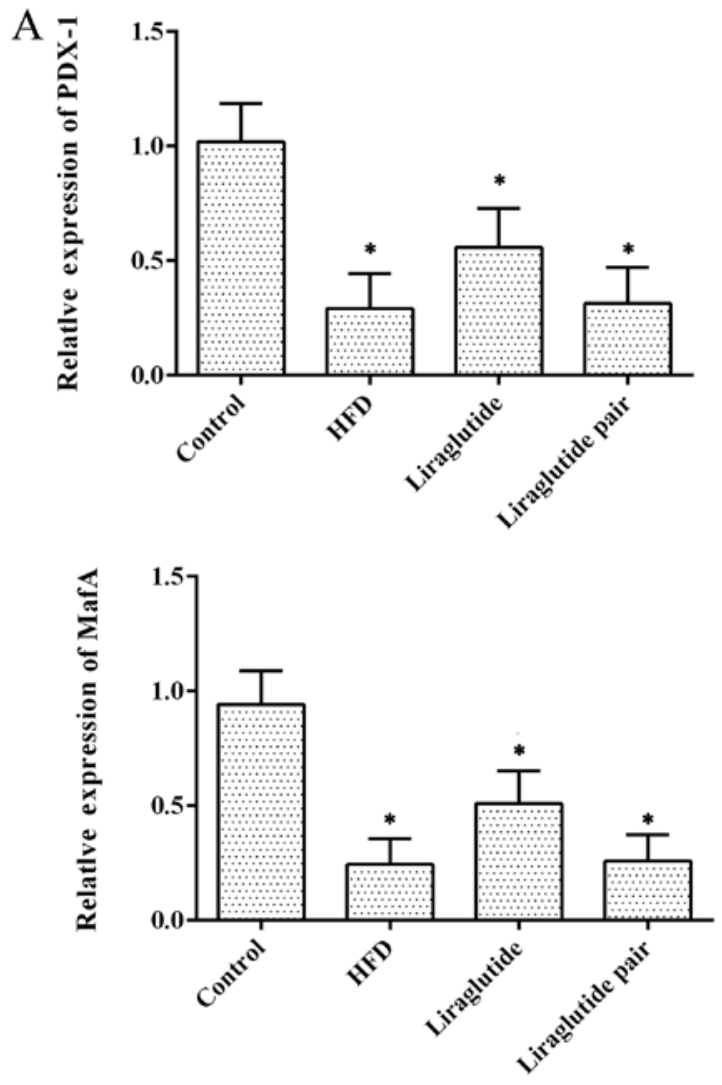

B

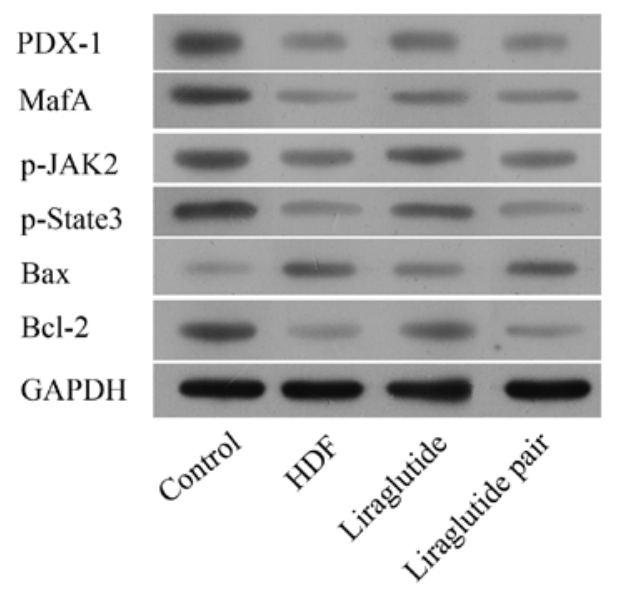

Figure 6. Liraglutide-induced activation of glucagon-like peptide 1 (GLP-1) exerts its function via activation of pancreatic and duodenal homeobox-1 (PDX-1). (A) Quantitative analysis results of RT-qPCR validation of expression of $P D X-1$ and MafA. The suppression of both indicators by high-fat diet (HFD) consumption was alleviated by administration of liraglutide. ${ }^{*} \mathrm{P}<0.05$, compared to the control group. (B) Representative images of western blotting of indicators related to pancreatic function. The expression levels of PDX-1, MafA, p-JAK2, p-State3 and Bcl-2 were supppressed by HFD consumption but activated by administration of liraglutide. The expression of Bax was induced by HFD but blocked by administration of liraglutide.

HFD and liraglutide paired groups (Fig. 5A) and no obvious proliferation of pancreas islet could be detected in the liraglutide group (Fig. 5A). Concomitant with the results of the H\&E staining, the apoptotic cell numbers in the HFD and liraglutide paired groups were also increased by HFD, and the apoptosis process was also inhibited by liraglutide treatment (Fig. 5B). FITC (TUNEL-positive) stains

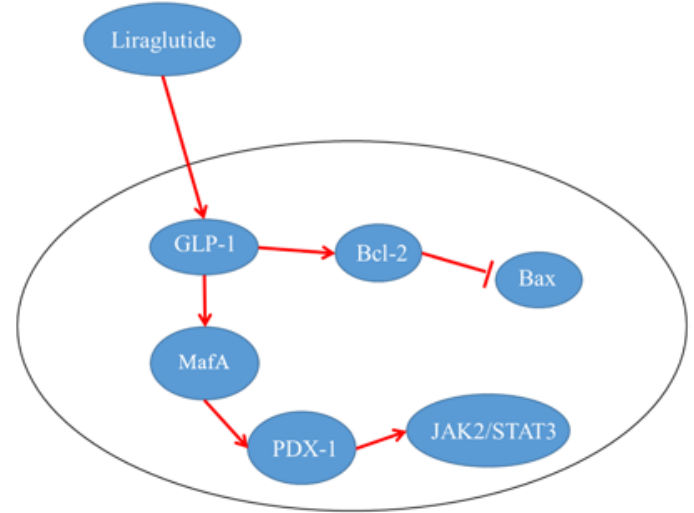

Figure 7. Regulatory sequencing involved in the protective effect of liraglutide on the pancreas.

green, Cy5 (insulin-positive) stains red, and DAPI stains blue. As illustrated by the immunofluorescent detection, islet $\beta$ cells (insulin-positive) were stained red and islet $\alpha$ cells (glucagan-positive) were stained green. In Fig. 5C, the number of islet $\beta$ cells (stained red) was reduced in the HFD and liraglutide paired groups compared with the control group, and administration of liraglutide increased the number of islet $\beta$ cells to a relatively normal level (Fig. 5C). In contrast, the number of islet $\alpha$ cells (stained green) was clearly increased by HFD and decreased by liraglutide administration, which was consistent with the augmentation of islet $\alpha$ cell function as shown above (Fig. 5C).

The protective effect of liraglutide-induced activation of GLP-1 is exerted through the activation of PDX-1. To further explore the mechanism through which GLP-1 exerts its function on the pancreas, the expression of PDX-1 was detected at both the mRNA and protein levels. As illustrated in Fig. 6A, at the mRNA level, expression of $P D X-1$ and MafA was inhibited in the HFD and liraglutide paired groups while they were significantly upregulated by liraglutide administration $(\mathrm{P}<0.05)$. Similar results were also detected at the protein level (Figs. 6B and 7). Such results offer a possible explanation that the pancreas protective effect of liraglutide-induced activation of GLP-1 may be exerted through the activation of PDX-1, which contributed to islet $\beta$ cell repair and insulin sensitivity. The protective effect of liraglutide was further confirmed by enhanced expression of MafA, p-JAK2 and p-Stat3, which indicated restoration of pancreatic function. Moreover, administration of liraglutide also clearly suppressed the expression of pro-apoptotic factor, Bax, compared with that noted in the HFD and liraglutide paired groups (Figs. 6B and 7) and increased the expression of anti-apoptotic factor Bcl-2, which corresponded to the anti-apoptotic effect of liraglutide as illustrated by TUNEL staining.

\section{Discussion}

Although a high-fat diet (HFD) is not common in all human populations, it is evidently recognized as a contributor to elevated blood triglyceride levels, which is validated as a risk factor for clinical acute pancreatitis, as are hyperlipidemia and obesity (28). In addition, HFD induced hyperinsulinemia and insulin resis- 
tance are two major characteristics of type 2 diabetes (1). An HFD has been proven to generate defective insulin secretion in animal models and can lead to fatty infiltration in the pancreas (29). Previous studies have demonstrated that HFD-induced insulin resistance in type 2 diabetes is attributed to the dysfunction and mass loss of islet $\beta$ cells (6-8). In the present study, HFD was employed to induce pancreatic injury and insulin resistance in C57/BL6 mice. Thereafter, the HFD-fed rats were administered liraglutide to ameliorate the impairments due to HFD. It was found that administration of liraglutide to HFD-fed mice restored the loss of islet $\beta$ cells and inhibited the proliferation of islet $\alpha$ cells, which contributed to the repair of pancreatic function. Moreover, detection at the molecular level indicated that the effect of liraglutide-induced activation of GLP-1 on pancreatic function may be exerted through the activation of PDX-1.

It has been acknowledged that GLP-1 suppresses glucagon release (30). Such suppression is independent of the increased insulin secretion in that it occurs even in cultured islet $\alpha$ cell lines (31). Furthermore, it has been reported that GLP-1 treatment stimulates islet $\beta$ cells without obvious changes in other cell types (32). In the present study, the treatment of HFD impaired islet $\beta$ cell function and induced delayed insulin release, islet $\beta$ cell apoptosis, and islet $\alpha$ cell hyperplasia in C57/ BL6 mice, representing the increased compensatory secretion in the pancreas. Such results were consistent with a previous study (33), confirming the successful induction of type 2 diabetes features in the present study. To reverse damage to the pancreas and islet $\beta$ cells, HFD-fed mice were subcutaneously injected with liraglutide, a type of GLP-1 receptor agonist. The administration of liraglutide significantly confounded the effect of HFD on the experimental animals. Firstly, the glucose tolerance of the mice in the liraglutide group was improved compared with that in the HFD group. Moreover, the damage to the structure and function of islet $\beta$ cells was clearly ameliorated, which could be further supported by the decreased insulin resistance in the hyperinsulinemic euglycemic clamp assay. Liraglutide treatment not only facilitated the function of recovery of islet $\beta$ cells but also inhibited the excess action of islet $\alpha$ cells. As shown in the liraglutide group, the number of islet $\alpha$ cells and secretion of GIP were both reduced by administration of liraglutide. Although the above results could provide compelling evidence of the effect of GLP-1 on HFD-induced pancreatic injury, the mechanism through which GLP-1 takes action was partially revealed.

Previous research (34) indicated that liraglutide administration markedly influences the body weight of the treated subjects. Whether the effect of liraglutide was exerted through this pattern should be comprehensively explored. To fulfill such purpose, a liraglutide paired group was established in the present study. Mice in this group were fed with the HFD as in the HFD group, but the calorie level was restricted to follow the weight trajectory of mice in the liraglutide group. By establishing the liraglutide paired group, it was found that although the decreased level of body weight could influence the results of some assays (difference between HFD and liraglutide paired groups was statistically insignificant), the overall effect of decreased body weight of the experimental mice had no impact on the outcomes of most experiments. Therefore, the effect of liraglutide-induced activation of GLP-1 was not attributed to its effect on the body weight of the experimental animals.
To further study the mechanism of GLP-1 in alleviating impairments due to HFD, the expression of PDX-1 was detected. PDX-1 is a critical regulator of islet $\beta$ cell growth and function in both fetal and developmental stages (13). Even a modest decrease in the release of the molecule can impair the compensatory response to insulin resistance $(16,35)$. In insulinoma cells and in islets from adult rodents, it stimulates insulin and somatostatin transcription $(36,37)$. It has been verified that development of type 2 diabetes is associated with progressive epigenetic silencing of $P D X-1$ (13). Thus, activation of PDX-1 may be a potential treatment strategy for prevention of type 2 diabetes. In the present study, administration of liraglutide contributed to the upregulation of PDX-1 both at the mRNA and protein level. The restoration of PDX-1 level was concomitant with the amelioration of the structure and function of the pancreas. The effect of GLP-1 on PDX-1 was previously revealed by Wang et al (38). The authors found that GLP-1 regulated the amount of PDX-1 in whole cell extracts and in nuclear extracts. Moreover, the effect of GLP-1 on nuclear PDX-1 was additive with glucose. Except for expression of PDX-1, levels of the other three markers of pancreatic function, including MafA, STAT3 and JAK2 were all enhanced by liraglutide (Fig. 7), which confirmed the protective effect of liraglutide-induced activation of GLP-1 on the pancreas $(26,27)$.

In conclusion, the findings outlined in the present study demonstrated that GLP-1 could ameliorate impairments on the pancreas due to HFD. Administration of GLP-1 receptor agonist reinforced the capacity of insulin to stimulate glucose disposal by restoring the function and number of islet $\beta$ cells, and inhibited the hyperplasia and redundant function of islet $\alpha$ cells. Moreover, the effect of GLP-1 was exerted through its activating effect on PDX-1 rather than its effect on a decrease in body weight, which supplemented the mechanism by which GLP-1 functions.

\section{References}

1. Knauf C, Cani PD, Ait-Belgnaoui A, Benani A, Dray C, Cabou C, Colom A, Uldry M, Rastrelli S, Sabatier E, et al: Brain glucagon-like peptide 1 signaling controls the onset of high-fat diet-induced insulin resistance and reduces energy expenditure. Endocrinology 149: 4768-4777, 2008.

2. Cummings DE and Schwartz MW: Genetics and pathophysiology of human obesity. Annu Rev Med 54: 453-471, 2003.

3. Alberti KG, Zimmet P and Shaw J; IDF Epidemiology Task Force Consensus Group: The metabolic syndrome - a new worldwide definition. Lancet 366: 1059-1062, 2005.

4. French SA, Story M and Jeffery RW: Environmental influences on eating and physical activity. Annu Rev Public Health 22: 309-335, 2001.

5. Wyatt SB, Winters KP and Dubbert PM: Overweight and obesity: prevalence, consequences, and causes of a growing public health problem. Am J Med Sci 331: 166-174, 2006.

6. Poitout V and Robertson RP: Minireview: secondary beta-cell failure in type 2 diabetes - a convergence of glucotoxicity and lipotoxicity. Endocrinology 143: 339-342, 2002.

7. Prentki M, Joly E, El-Assaad W and Roduit R: Malonyl-CoA signaling, lipid partitioning, and glucolipotoxicity: role in $\beta$-cell adaptation and failure in the etiology of diabetes. Diabetes 51 (Suppl 3): S405-S413, 2002.

8. Kaiser N, Leibowitz G and Nesher R: Glucotoxicity and beta-cell failure in type 2 diabetes mellitus. J Pediatr Endocrinol Metab 16: 5-22, 2003.

9. Nakae J, Biggs WH III, Kitamura T, Cavenee WK, Wright CV, Arden KC and Accili D: Regulation of insulin action and pancreatic beta-cell function by mutated alleles of the gene encoding forkhead transcription factor Foxo1. Nat Genet 32: 245-253, 2002. 
10. Sussel L, Kalamaras J, Hartigan-O'Connor DJ, Meneses JJ, Pedersen RA, Rubenstein JL and German MS: Mice lacking the homeodomain transcription factor $\mathrm{Nkx} 2.2$ have diabetes due to arrested differentiation of pancreatic beta cells. Development 125: 2213-2221, 1998.

11. Withers DJ, Burks DJ, Towery HH, Altamuro SL, Flint CL and White MF: Irs-2 coordinates Igf-1 receptor-mediated beta-cell development and peripheral insulin signalling. Nat Genet 23: 32-40, 1999.

12. Offield MF, Jetton TL, Labosky PA, Ray M, Stein RW, Magnuson MA, Hogan BL and Wright CV: PDX-1 is required for pancreatic outgrowth and differentiation of the rostral duodenum. Development 122: 983-995, 1996.

13. Park JH, Stoffers DA, Nicholls RD and Simmons RA: Development of type 2 diabetes following intrauterine growth retardation in rats is associated with progressive epigenetic silencing of Pdx1. J Clin Invest 118: 2316-2324, 2008.

14. Brissova M, Blaha M, Spear C, Nicholson W, Radhika A, Shiota M, Charron MJ, Wright CV and Powers AC: Reduced PDX-1 expression impairs islet response to insulin resistance and worsens glucose homeostasis. Am J Physiol Endocrinol Metab 288: E707-E714, 2005.

15. Kulkarni RN, Jhala US, Winnay JN, Krajewski S, Montminy M and Kahn CR: PDX-1 haploinsufficiency limits the compensatory islet hyperplasia that occurs in response to insulin resistance. $\mathrm{J}$ Clin Invest 114: 828-836, 2004.

16. Holland AM, Góñez LJ, Naselli G, Macdonald RJ and Harrison LC: Conditional expression demonstrates the role of the homeodomain transcription factor Pdx1 in maintenance and regeneration of beta-cells in the adult pancreas. Diabetes 54: 2586-2595, 2005.

17. Guz Y, Montminy MR, Stein R, Leonard J, Gamer LW, Wright CV and Teitelman G: Expression of murine STF-1, a putative insulin gene transcription factor, in beta cells of pancreas, duodenal epithelium and pancreatic exocrine and endocrine progenitors during ontogeny. Development 121: 11-18, 1995.

18. Peshavaria M, Gamer L, Henderson E, Teitelman G, Wright CV and Stein R: XIHbox 8, an endoderm-specific Xenopus homeodomain protein, is closely related to a mammalian insulin gene transcription factor. Mol Endocrinol 8: 806-816, 1994.

19. Stoffers DA, Desai BM, DeLeon DD and Simmons RA: Neonatal exendin-4 prevents the development of diabetes in the intrauterine growth retarded rat. Diabetes 52: 734-740, 2003

20. Holst JJ: Glucagonlike peptide 1: a newly discovered gastrointestinal hormone. Gastroenterology 107: 1848-1855, 1994.

21. Holst JJ, Ørskov C, Nielsen OV and Schwartz TW: Truncated glucagon-like peptide I, an insulin-releasing hormone from the distal gut. FEBS Lett 211: 169-174, 1987.

22. Kreymann B, Williams G, Ghatei MA and Bloom SR: Glucagon-like peptide-1 7-36: a physiological incretin in man. Lancet 2: 1300-1304, 1987.

23. Flint A, Raben A, Astrup A and Holst JJ: Glucagon-like peptide 1 promotes satiety and suppresses energy intake in humans. J Clin Invest 101: 515-520, 1998.

24. Gutzwiller JP, Göke B, Drewe J, Hildebrand P, Ketterer S, Handschin D, Winterhalder R, Conen D and Beglinger C: Glucagon-like peptide-1 is a physiologic regulator of food intake in humans. Gut 44: 81-86, 1999.

25. Parlevliet ET, de Leeuw van Weenen JE, Romijn JA and Pijl $\mathrm{H}$ : GLP-1 treatment reduces endogenous insulin resistance via activation of central GLP-1 receptors in mice fed a high-fat diet. Am J Physiol Endocrinol Metab 299: E318-E324, 2010.
26. Zhang C, Moriguchi T, Kajihara M, Esaki R, Harada A, Shimohata H, Oishi H, Hamada M, Morito N, Hasegawa K, et al: MafA is a key regulator of glucose-stimulated insulin secretion. Mol Cell Biol 25: 4969-4976, 2005.

27. Corcoran RB, Contino G, Deshpande V, Tzatsos A, Conrad C, Benes CH, Levy DE, Settleman J, Engelman JA and Bardeesy N: STAT3 plays a critical role in KRAS-induced pancreatic tumorigenesis. Cancer Res 71: 5020-5029, 2011.

28. Tsuang W, Navaneethan U, Ruiz L, Palascak JB and Gelrud A: Hypertriglyceridemic pancreatitis: presentation and management. Am J Gastroenterol 104: 984-991, 2009.

29. Cedernaes J, Alsiö J, Västermark A, Risérus U and Schiöth HB: Adipose tissue stearoyl-CoA desaturase 1 index is increased and linoleic acid is decreased in obesity-prone rats fed a high-fat diet. Lipids Health Dis 12: 2, 2013.

30. Ritzel R, Orskov C, Holst JJ and Nauck MA: Pharmacokinetic, insulinotropic, and glucagonostatic properties of GLP-1 [7-36 amide] after subcutaneous injection in healthy volunteers. Dose-response-relationships. Diabetologia 38: 720-725, 1995.

31. Matsumura T, Itoh H, Watanabe N, Oda Y, Tanaka M, Namba M, Kono N, Matsuyama T, Komatsu R and Matsuzawa Y: Glucagonlike peptide-1(7-36)amide suppresses glucagon secretion and decreases cyclic AMP concentration in cultured In-R1-G9 cells. Biochem Biophys Res Commun 186: 503-508, 1992.

32. Friedrichsen BN, Neubauer N, Lee YC, Gram VK, Blume N, Petersen JS, Nielsen JH and Møldrup A: Stimulation of pancreatic beta-cell replication by incretins involves transcriptional induction of cyclin D1 via multiple signalling pathways. J Endocrinol 188: 481-492, 2006.

33. Schwasinger-Schmidt T, Robbins DC, Williams SJ, Novikova L and Stehno-Bittel L: Long-term liraglutide treatment is associated with increased insulin content and secretion in $\beta$-cells, and a loss of $\alpha$-cells in ZDF rats. Pharmacol Res 76: 58-66, 2013

34. Nauck MA, Hompesch M, Filipczak R, Le TD, Zdravkovic M and Gumprecht J; NN2211-1499 Study Group: Five weeks of treatment with the GLP-1 analogue liraglutide improves glycaemic control and lowers body weight in subjects with type 2 diabetes. Exp Clin Endocrinol Diabetes 114: 417-423, 2006.

35. Del Guerra S, Lupi R, Marselli L, Masini M, Bugliani M, Sbrana S, Torri S, Pollera M, Boggi U, Mosca F, et al: Functional and molecular defects of pancreatic islets in human type 2 diabetes. Diabetes 54: 727-735, 2005.

36. Petersen HV, Serup P, Leonard J, Michelsen BK and Madsen OD: Transcriptional regulation of the human insulin gene is dependent on the homeodomain protein STF1/IPF1 acting through the CT boxes. Proc Natl Acad Sci USA 91: 10465-10469, 1994.

37. Miller CP, McGehee RE Jr and Habener JF: IDX-1: a new homeodomain transcription factor expressed in rat pancreatic islets and duodenum that transactivates the somatostatin gene. EMBO J 13: 1145-1156, 1994.

38. Wang X, Cahill CM, Piñeyro MA, Zhou J, Doyle ME and Egan JM: Glucagon-like peptide-1 regulates the beta cell transcription factor, PDX-1, in insulinoma cells. Endocrinology 140: 4904-4907, 1999. 